\title{
The Role of Episodic Buffer in Episodic Future Thinking
}

\author{
Rumana Ferdousi Siddique ${ }^{1, *}$, Asheek Mohammad Shimul ${ }^{2}$, Alim Ul Gias ${ }^{3}$, Mohayeminul Islam ${ }^{3}$, \\ Shah Mostafa Khaled ${ }^{3}$ \\ ${ }^{1}$ Dhaka University Memory Research Unit (DUMRU), Department of Psychology, University of Dhaka, Bangladesh \\ ${ }^{2}$ Dhaka University Memory Research Unit, Department of Psychology, University of Dhaka, Bangladesh \\ ${ }^{3}$ Institute of Information Technology, University of Dhaka, Bangladesh
}

Copyright $@ 2015$ Horizon Research Publishing All rights reserved.

\begin{abstract}
Present research attempted to see the role performed by episodic buffer in the generation of future thinking by making episodic buffer engage in dual task while generating future thoughts. Hypothesis anticipated differences in response accuracy and required time of response. Participants performed episodic buffer task of verbal-spatial binding while retrieving events of past and constructing events of future. While comparing past and future retrieval, variances in response accuracy and reaction time was not evident but memory specificity appeared noteworthy. In addition, participants' observer or third person perspective facilitated to generate more specific memory which is inconsistent with previous researches [20] [21] [23] that lead to the necessity of more extensive enquiries. As concerns episodic buffer, present study suggests no further significant contribution of buffer in future thinking than thinking about the past.
\end{abstract}

Keywords Episodic Buffer, Dual Task, Verbal-spatial Binding, Episodic Future Thinking

\section{Introduction}

Human mind's distinctive ability of mentally simulate imagined future events has been extensively researched in recent years both theoretically and empirically [26] [34]. Episodic memory and future thought are often thought to serve as complementary functions and are together viewed as comprising the broader faculty of mental time travel [30]. Memories of past events and foresight into the future are fundamental in creating the phenomenological representation of self-concept that is consistent across space and time [36].

For a number of years the ability to imagine hypothetical events in one's personal future or "episodic future thinking" [4] has been argued to be closely related to the ability to remember one's past experiences [30] [38]. Much like episodic memory, episodic future thought is believed to be a constructive process in which distinct pieces of information are retrieved from long-term memory and flexibly recombined into a coherent imagined event [24]. Converging evidence from a variety of research domains lends support for this view.

Behavioral study by Szpunar \& McDermott [32] indicated that the contents of episodic memory are repeatedly sampled during the construction of future scenarios. Developmental studies provide further evidence that though children begin to successfully recall previously experienced events at a younger age, but it is not until the age of about four that they begin to generate contextually rich and personally significant episodic memories [16] [29] [38]. Study of Addis, Wong, \& Schacter [2] revealed that, age related declines in episodic memory correspond with similar impairments in future oriented thought.

Brain imaging evidences have indicated a core neural network underlying episodic memory and future thought, including areas of the medial prefrontal cortex (mPFC), medial temporal lobes (MTL), and extending posteriorly into the parietal and occipital lobes [1] [2] [7] [22] [31]. Neuropsychological Evidences of schizophrenic [9] , amnesiac [13] and autistic adults [19] reported trouble in generating specific past and future events relative to comparison populations, suggesting some common cognitive mechanism.

All these studies demonstrate a close relationship between remembering past events and imagining future events and explored that our ability to imagine or simulate future events relies on multiple component processes (including working memory, executive control processes, relational processes, visual-spatial processing, self-consciousness, and the apprehension of subjective time), most of which are also involved in remembering past episodes [10]. Most of the processes underlying future thinking are controlled by the components of working memory [10], but Hill \& Emery [14] suggested that when imagining future events, working memory contributes to the construction of a single, coherent, 
future event illustration, but not to the retrieval or elaboration of event details.

Recently episodic buffer, component of WM, has been suggested to act as the "stage" on which episodic details are recombined into a hypothetical scenario [25] [30] as it is thought to store bound multimodal constructions kept in consciousness through interaction with the central executive. This is consistent with the idea that imagining the future involves combining information from multiple modalities, including past experiences, visualization processes [33] [31], and semantic personal information [11] [37].

As it is well established in memory literature that episodic buffer provides the appropriate medium for linking the phonological loop and visuo-spatial representations with those from long-term memory, and that the central executive control the allocation of information from different sources into the buffer [5], it might have a unique role in future thinking. But recent findings are inconsistent with the suggestion that feature binding takes place in the episodic buffer [5] and support that it takes place automatically before entering the episodic buffer [6]. So, what specific role is performed by episodic buffer in future thinking has remained uninvestigated. Expanding on this idea current study investigated the relationship between episodic future thinking and episodic buffer.

Another important aspect of concern was persons adopted view about the retrieval of past memory and generation of possible future memory. Events of the personal past are retrievable from two perspectives. One is through the rememberer's own eye; another is from a spectator's standpoint. As the applicability of person's point of view has been investigated for episodic memory retrieval but not in case of future thinking which led us to enquire systematically and see the differences. Hypotheses that have been formed to test were- response accuracy, reaction time and memory specificity would differ between past and future conditions; there would be a gender effect in reaction time and finally an effect of viewpoint would be in specific memory retrieval.

\section{Method}

\subsection{Participants}

A total of 60 undergraduate volunteers took part in the 30 -min study. Among them, 30 were male (50\%) and 30 were female $(50 \%)$. Participants' age ranges from 17-22 years with mean age 19.5 years. All participants reported normal or corrected-to-normal vision, and all were naive to the experimental aims. All of them were healthy adult participants who met the criteria of not having any sort of memory problems.

\subsection{Materials}

Stimuli were presented on a 10 inch computer screen. The task was purpose written using Work Memory Test Software.
The verbal stimuli comprised a set of eight upper-case consonants, and were: D; F; H; J; N; Q; R; T. The spatial stimuli were presented within a set of eight spatial locations placed randomly. Participants had to complete verbal memory retrieval task while performing episodic buffer task (verbal-spatial binding). A voice recorder recorded the memory recollection of both past and future condition. A response sheet was used to identify the point of view of the participants during memory recollection. It requires responding either as an observer or as an active participant who was presented in the field of action.

\subsection{Experimental Design and Procedure}

There were three distinct conditions- baseline, past and future. Each of these conditions (except baseline) consists of three different levels named phases. Participants had to go through total eight levels- practice, baseline, past 1, past 2, past 3 , future 1 , future 2 , and future 3 . Here the baseline was no task condition; past condition 1,2 and 3 were characterized by the concurrent task with retrieving memory of 10 years, 6 months and 1 month old respectively and future 1, 2 and 3 conditions were characterized by generating events that would occur after 1 month, 6 months and 10 years later along with the concurrent task. Each phase continued for $1 \mathrm{~min}$. In the first two phases, participants responded only to the episodic buffer task (computer task) and in other phases they retrieved/generated memory (past/ future) along with the episodic buffer task. On each trial participants were presented with a TBR array (To be remembered array), a visual mask (a black \& white hazy picture), a blank screen and finally a probe item. The TBR array consisted of four black consonants (against a white background) selected at random (without replacement) from the set of eight (so that no letter was repeated in each set of four). Each trial began with the presentation of a ready "+" signal (black, Arial font; $18 \mathrm{pt}$ ) for $500 \mathrm{~ms}$, followed by the presentation of a to-be-remembered array of consonants in locations $(2,000$ $\mathrm{ms}$ ). A $50 \mathrm{~ms}$ visual mask then replaced the array. A single probe item was then presented. The participants' task was to indicate, by pressing keyboard keys (" $\mathrm{z}$ " for "YES", " $n$ " for "NO"), whether a probe item represented both a letter and a location that was present in the TBR array (see Figure 1 for a schematic illustration of a trial).

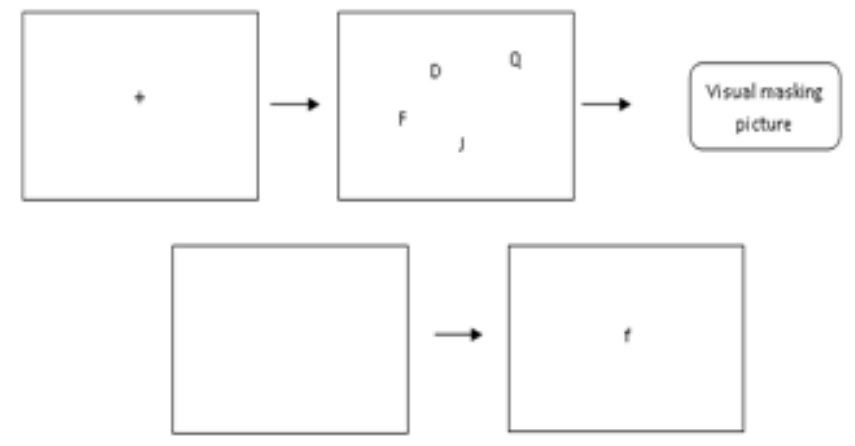

Figure 1. Schematic illustration of a single trial in the verbal condition. 
After the completion of practice and baseline condition, the concurrent task condition began where the participants had to perform two tasks at a time. The concurrent task condition was divided into two distinct conditions, one was past condition and the other was future condition. The conditions were counterbalanced for each participant. Each of these conditions again has three other levels defined by three different time limits. Participants were asked to describe a specific memorable event as specifically as possible (i.e., unique event that took place in a specific place at a specific time and that lasted for few minutes or hours, but not more than a day) which was occurred in his/her life before 10 years, 6 months and 1 month period respectively. Participants were asked to provide a detailed description of events that were personally experienced while performing episodic buffer task.

After the concurrent task of each phase, participants were provided with a viewpoint response sheet. This response sheet was about the viewpoint or perspective that the person held during the recollection of memory. They responded by providing tick $(\sqrt{ })$ to either field view or observer view. After the past condition, next and last condition was the future memory retrieval condition. It also had three distinct levels of different time periods. Participants were asked to describe specific memorable events (i.e., unique events that would take place in a specific place at a specific time and would last for few minutes or hours, but not more than a day) which would occur in his/her life after 1 month, 6 months and 10 years period respectively. The recollections of both past and future events were recorded in the voice-recorder and the responses concerning viewpoint were taken.

\subsection{Scoring}

Table 1. Autobiographical Interview Scoring Categories

\begin{tabular}{ccl}
\hline $\begin{array}{c}\text { Detail } \\
\text { Type }\end{array}$ & Detail Category & \multicolumn{1}{c}{ Description } \\
\hline Internal & Event & $\begin{array}{l}\text { Happenings, individual's present, } \\
\text { weather, physical/emotional } \\
\text { actions/reactions in others. } \\
\text { Year, season, month, day of } \\
\text { week, time of day } \\
\text { Localization of an event } \\
\text { including the city, street, } \\
\text { building, room, part of room } \\
\text { Auditory, olfactory, tactile, taste, } \\
\text { visual and visual details, body } \\
\text { position, duration } \\
\text { Emotional state, thoughts, } \\
\text { implications. }\end{array}$ \\
\hline
\end{tabular}

Data analysis started with data verbatim transcription (verbal to written). Each memory description was divided into different informational segments or details. A detail was defined as a unique occurrence, observation, or thought. Each detail was then coded according to the standardized Autobiographical Interview procedure outlined in Levine et al. [17] [18]. Details were defined as "internal" or episodic and allocated to one of the five categories (event, place, time, perceptual, and emotion/thought) if they related directly to the main event described, specific in time and place, of limited duration (i.e., a few hours), and express a sense of episodic re-experiencing. Otherwise, details were excluded from analysis.

The excluded details were semantic memory (factual information), events that did not occurred in a specific time and place, repetition of information and other metacognitive statements. Each specific detail was identified as one specific memory item and the cumulative of all the specific items that recollected in each memory retrieval level was the number of memory specificity for that level (10 years before/6 months after etc.) of particular condition (past/future). One of the examples of scoring process according to the standardized Autobiographical Interview Procedure outlined in Levine [18] is given below.

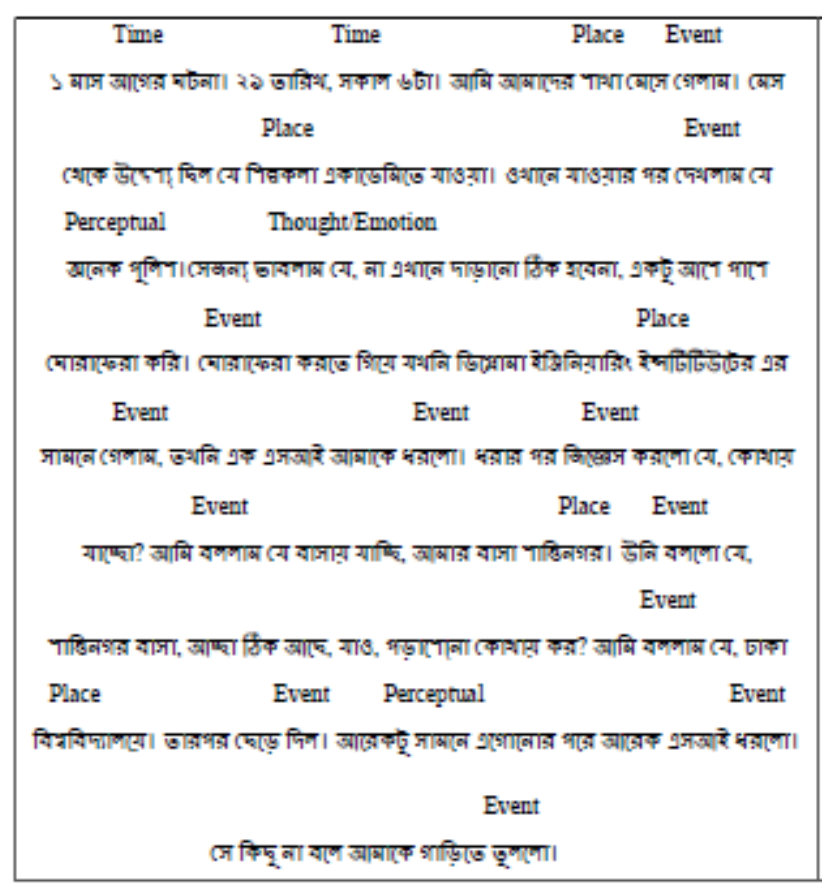

Figure 2. Scoring example using Autobiographical Memory Interview standardized coding. Event, time, place, perceptual, and thought/emotion are classified as the internal detail type.

\section{Results}

In analyzing the data, descriptive statistics, paired t-test, independent sample $t$ - test and two-way ANOVA were used to test all the hypotheses. Besides, Mauchly's test was used to test the assumption of sphericity. Greenhouse-Geisser correction was used to adjust for violations in the repeated measure assumptions for the latter analyses. An alpha level of 0.05 was used for all statistical tests.

\subsection{Average Reaction Time}

Table 2 presents mean difference of average reaction time of past and future condition. Results indicated no significant difference between the average reaction time of 
past condition and future condition.

Table 2. Paired Samples Test for Average Reaction Time of Responses for Past Condition and Future Condition

\begin{tabular}{ccccc}
\hline Time & $M$ & $S D$ & $t$ & Sig.(2-tailed) \\
\hline Past & 3642.2 & 2940.5 & & \\
Future & 3440.9 & 2416.9 & 0.592 & 0.556 \\
\hline
\end{tabular}

Table 3. Summary of ANOVA for Average Reaction Time of Past and Future Condition

\begin{tabular}{cccc}
\hline Variable & $d f$ & $F$ & Sig. \\
\hline Time & 1 & 0.351 & 0.56 \\
Phase & 59 & 3.049 & $0.05 *$ \\
Time*Phase & 2 & & \\
$*_{p} \leq 0.05$ & 118 & 3.152 & $0.04 *$ \\
\hline
\end{tabular}

Table 3 indicated that the main effect of phases $[F(2,118)$ $=3.05, p \leq .05]$ and interaction effects between time and phases $[F(2,118)=3.15, p \leq .05]$ were significant. Again, results showed that main effect of time conditions was not significant for average reaction time, $[F(1,59)=.35$, $p>.05]$.

Table 4 indicates the mean differences of average reaction time for both past and future conditions by gender. There was no significant difference in the mean reaction time of past condition between male and female participants but the average reaction time in future condition differed significantly between male and female participants. Results partially accepted the hypothesis that there was a gender effect on the average reaction time of response.

Table 4. Mean Differences of Average Reaction Time of Past and Future Condition for Male and Female Participants

\begin{tabular}{ccccccc}
\hline Time & Gender & $N$ & $M$ & $S D$ & $t$ & $\begin{array}{c}\text { Sig. } \\
\text { (2-tailed) }\end{array}$ \\
\hline \multirow{2}{*}{ Past } & Male & 30 & 3575.6 & 1843.4 & 0.174 & 0.86 \\
& Female & 30 & 3708.8 & 3766.2 & & \\
\hline \multirow{2}{*}{ Future } & Male & 30 & 4106.9 & 2998.3 & 2.203 & $0.03 *$ \\
& Female & 30 & 2774.8 & 1405.8 & &
\end{tabular}

Table 5 indicates the differences of average reaction time for the phases of past and future conditions according to gender. No significant difference was found in the mean reaction time for the phases of past condition between male and female participants. This result was also evident for the phase 1 of future condition but the average reaction time in phase 2 and phase 3 of future condition differed significantly between male and female participants.

Table 6 indicated that the main effect of phases $[F(2$, $116)=3.75, p \leq .05]$, interaction effects between time and gender $[F(2,116)=4.96, \mathrm{p} \leq .05]$ and interaction effects of phase and gender $[F(2,116)=3.00, \mathrm{p} \leq .05]$ were significant. Again, results revealed that main effect of time conditions, interaction effects of time and phase, and interaction of time, phase and gender were not significant for average reaction time.

Table 5. Mean Differences of Average Reaction Time of Phases for Male and Female Participants

\begin{tabular}{|c|c|c|c|c|c|c|c|}
\hline Time & Phases & Gender & $N$ & $M$ & $S D$ & $T$ & Sig.(2-tailed) \\
\hline \multirow{3}{*}{$\begin{array}{c}\text { Past } \\
\text { Condition }\end{array}$} & Phase 1 & $\begin{array}{l}\text { Male } \\
\text { Female }\end{array}$ & $\begin{array}{l}30 \\
30\end{array}$ & $\begin{array}{l}3844.86 \\
5576.01\end{array}$ & $\begin{array}{l}2524.86 \\
9664.18\end{array}$ & 0.95 & 0.34 \\
\hline & Phase 2 & $\begin{array}{c}\text { Male } \\
\text { Female }\end{array}$ & $\begin{array}{l}30 \\
30\end{array}$ & $\begin{array}{l}3615.67 \\
3023.99\end{array}$ & $\begin{array}{l}2166.58 \\
1636.74\end{array}$ & 1.19 & 0.23 \\
\hline & Phase 3 & $\begin{array}{c}\text { Male } \\
\text { Female }\end{array}$ & $\begin{array}{l}30 \\
30\end{array}$ & $\begin{array}{l}3266.17 \\
2526.27\end{array}$ & $\begin{array}{l}2121.42 \\
1731.92\end{array}$ & 1.48 & 0.14 \\
\hline \multirow{3}{*}{$\begin{array}{l}\text { Future } \\
\text { Condition }\end{array}$} & Phase 1 & $\begin{array}{c}\text { Male } \\
\text { Female }\end{array}$ & $\begin{array}{l}30 \\
30\end{array}$ & $\begin{array}{l}3894.21 \\
3089.87\end{array}$ & $\begin{array}{l}2777.57 \\
1636.09\end{array}$ & 1.37 & 0.17 \\
\hline & Phase 2 & $\begin{array}{c}\text { Male } \\
\text { Female }\end{array}$ & $\begin{array}{l}30 \\
30\end{array}$ & \begin{tabular}{|l}
3889.19 \\
2615.02 \\
\end{tabular} & $\begin{array}{l}3018.67 \\
1410.49 \\
\end{array}$ & 2.09 & $0.04 *$ \\
\hline & Phase 3 & $\begin{array}{c}\text { Male } \\
\text { Female }\end{array}$ & $\begin{array}{l}30 \\
30\end{array}$ & $\begin{array}{l}4537.46 \\
2619.59 \\
\end{array}$ & $\begin{array}{l}4173.31 \\
1731.68 \\
\end{array}$ & 2.32 & $0.02 *$ \\
\hline
\end{tabular}

$* p<0.05$

Table 6. Summary of ANOVA for Average Reaction Time of Past and Future Condition between Male and Female

\begin{tabular}{cccc}
\hline Variable & $d f$ & $F$ & Sig. \\
\hline Time & 1 & 0.37 & 0.54 \\
Time*Gender & 58 & 4.96 & $0.03^{*}$ \\
Phase & 1 & 3.75 & $0.02^{*}$ \\
Phase*Gender & 58 & 3.0 & $0.05^{*}$ \\
Time*Phase & 2 & 2.56 & 0.08 \\
Time*Phase*Gender & 116 & 0.83 & 0.43 \\
\hline
\end{tabular}




\subsection{Correct Response}

Table 7 shows the mean difference of correct response between past and future condition. It was evident from result that there was no significant difference between the correct response of past condition and future condition.

Table 7. Paired Samples Test for Correct Response of Past Condition and Future Condition

\begin{tabular}{cccccc}
\hline Conditions & $M$ & $S D$ & $t$ & $d f$ & Sig.(2-tailed) \\
\hline Past & 7.98 & 3.07 & 0.533 & 59 & 0.596 \\
Future & 8.14 & 3.38 & & \\
\hline
\end{tabular}

Table 8. Summary of ANOVA for Correct Response of Past and Future Condition

\begin{tabular}{cccc}
\hline Variables & $d f$ & $F$ & Sig. \\
\hline Time & 1 & 0.284 & 0.59 \\
Phase & 59 & & \\
& 2 & 3.71 & $0.02 *$ \\
Time*Phase & 118 & & \\
* $_{\mathrm{p}<0.05}$ & 118 & 0.785 & 0.45 \\
\hline
\end{tabular}

$* \mathrm{p} \leq 0.05$

Using two-way ANOVA, table 8 indicated that there was a significant effect of phases in the difference of correct response $\left[F_{(2,118)}=3.71, p<.05\right]$. But, both the effect of time $\left[F_{(1,59)}=.284, p>.05\right]$ and the interaction effect between time and phase $\left[F_{(2,118)}=.785, p>.05\right]$ were non-significant for correct response. That is correct response considerably differed among the phases of different conditions.

\subsection{Memory Specificity}

Table 9 indicated that a significant difference was found between the memory specificity of past condition and future condition. Results accepted the hypothesis that considerable difference was present in the specific memory retrieval between past and future conditions.

Table 9. Paired Samples Test for Memory Specificity of Past Condition and Future Condition

\begin{tabular}{ccccc}
\hline Conditions & $M$ & $S D$ & $t$ & $\begin{array}{c}\text { Sig. } \\
\text { (2- tailed) }\end{array}$ \\
\hline Past & 16.36 & 3.18 & 8.39 & $0.000^{*}$ \\
Future & 12.53 & 3.58 & &
\end{tabular}

Using two-way ANOVA, it was evident from table 10 that there was a significant effect of time $\left[F_{(1,59)}=70.41, p<.05\right]$ for memory specificity. But, both the effect of phase $\left[F_{(2,118)}\right.$ $=.184, p>.05]$ and the interaction effect between time and phase $\left[F_{(2,118)}=2.92, p>.05\right]$ were non-significant for memory specificity.

Table 10. Summary of ANOVA for Memory Specificity of Past and Future Condition

\begin{tabular}{cccc}
\hline Variables & $d f$ & $F$ & Sig. \\
\hline Time & 1 & 70.41 & $.000^{*}$ \\
Phase & 59 & & \\
& 2 & 0.184 & 0.82 \\
Time*Phase & 2 & & \\
\hline
\end{tabular}

$$
* p \leq 0.05
$$

Figure 3 illustrates the effects of participants' viewpoint on the specific memory retrieval in both past and future conditions. In the first two levels of past condition, observer view facilitated the retrieval where field view facilitated only on third level. During future condition, observer view superimposed field view for the better memory recall. It can be concluded that viewpoint had effects on memory specificity but it did not reveal any consistent relation in respect of time except the recommendation that observer view enhance the ability to be more specific in the memory regeneration.

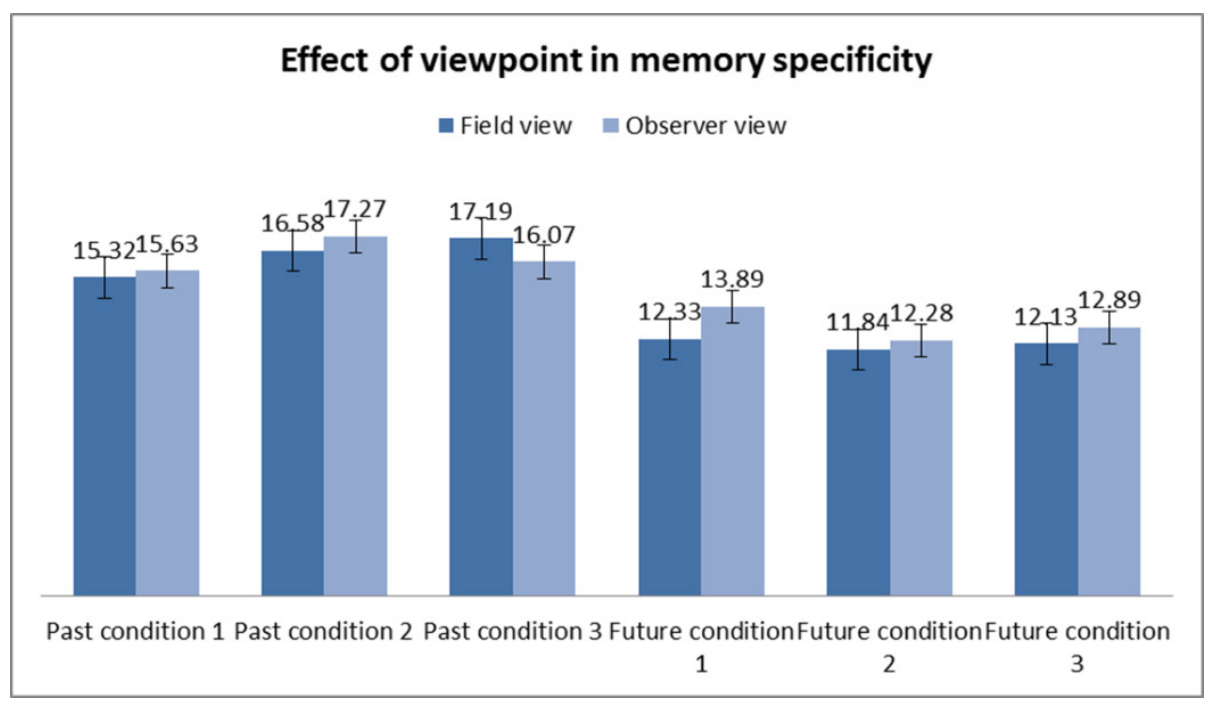

Figure 3. Mean of Specific Memory in Different Viewpoint 


\section{Discussion}

In the present study individual differences approach was taken to investigate the role episodic buffer plays in the ability to construct future episodic events. Participants completed tasks as measures of episodic memory retrieval and future thought generation with concurrent visuo-spatial binding of the episodic buffer task. Hypotheses were to see whether any difference do exist or not between past memory retrieval and future thought generation in terms of reaction time, correct response and memory specificity of episodic buffer task. The researchers also investigated the gender effect on average reaction time and whether there is any impact of viewpoint in the memory construction. Results indicates that: (a) as measures of episodic buffer contribution, response accuracy and reaction time of response do not differ between past memory regeneration and episodic future thinking, but a gender effect is evident for future reaction time of response; and (b) memory specificity is significantly higher for past retrieval than in future thinking. It is also found that (c) third-person perspective or observer view of participants' facilitate specific memory retrieval.

Present study is unable to provide support concerning the difference in demand of episodic buffer task during past and future memory retrieval. It reveals that response accuracy and reaction time as measures of episodic buffer are lesser for baseline condition compared to concurrent task conditions which conforms previous studies but not found significant between past and future. As it is evident from previous researches that construction of future memory is more executively challenging but the results found in the present study is contradictory which find support from the previous researches that when simulating future events, individuals might simply recast a memory of a past experience as an imagined future event that might led to similar consequences as past retrieval does. Previous findings of greater activation of the core network when simulating future events [2] [31] could be taken to speak against this possibility, suggesting that constructing future events is more cognitively demanding process requiring the recombination of details. However, it is also possible that the process of 'recasting' a past event as a future event, rather than simply retrieving it as required in the past condition, also results in similar consequence [1]. There is also a gender effect evident on future mean reaction time of response. Male participants are found to take more time to response than female during future memory construction. Similar difference is not found in the past condition. It is well-established in memory literature that males and females differ significantly in their cognitive abilities. In case of spatial visualization and mental rotation which are measures of prospection, boys outperforms girls and the tasks that require retrieval from long-term memory and the acquisition and use of verbal information show a female advantage. Females show higher levels of ability on a variety of memory tasks [28]. Stumpf and Eliot [27] examined academically talented students and found an advantage for females, this time on tests of visual memory [12]. Girls were found to imagine their near future in familiar contexts such as home environment, work place etc., on the other hand boys were found to imagine themselves in more diverse situations in near or far future. This observation leads to the conclusion that imagining themselves in diverse situation caused males to take more reaction time to response. So, this finding may also give rise to a hypothesis that male-female differ in memory retrieval and subsequently memory generation with concurrent activity and females are more imaginative than males concerning their future that require further extensive research.

Memory specificity, third variable of concern, is found significantly different between past and future conditions. This finding conform the previous research findings [14] [8] [10] that, accessing past information appears to load on largely overlapping retrieval mechanisms. Finally, participants' viewpoint do not show any significant consistent relationship but give rise to a hypothesis that persons' adopted observer view during memory retrieval facilitate memory specificity as among three distinct levels of both past and future conditions, participants produced more specific memory in two levels of each condition. Evidence from several behavioral sources suggests that memories relived from a field or first-person perspective have an increased level of subjective emotionality, relative to memories retrieved from an observer or third-person perspective [20] [21] [23] which lead to more specific memory retrieval of past events. Memories recalled from a first-person "field" perspective are phenomenologically rich, while third-person "observer" perspective memories contain more descriptive but less affective detail [21]. So, in case of present study participants' adoption of observer view might led to the retrieval of more descriptive details, thus facilitated memory specificity. So, conclusion can be drawn as the role of episodic buffer in episodic future thinking does not significantly differ than in regenerating past. In addition, effects of visual perspective lead to a conclusion that suggests further detailed research.

\section{REFERENCES}

[1] Addis, D., Pan, L., Vu, M., Laiser, N., \& Schacter, D. (2008). Constructive episodic simulation of the future and the past: Distinct subsystems of a core brain network mediate imagining and remembering. Neuropsychologia, 47, 2222-2238. doi:10.1016/j.neuropsychologia.2008.10.026

[2] Addis, D., Wong, A., \& Schacter, D. (2007). Remembering the past and imagining the future: Common and distinct neural substrates during event construction and elaboration. Neuropsychologia, 1363-1377. doi:10.1016/j.neuropsychologia.2006.10.016

[3] Atance, C. M., \& O’Neill, D. K. (2001). Episodic future thinking. TRENDS in Cognitive Sciences, 5, 533-539. 
doi:10.1016/S1364-6613(00)01804-0

[4] Atance, C. M., \& O'Neill, D. K. (2005). The emergence of episodic future thinking in humans. Learn. Motiv., 36, 126144.

[5] Baddeley, A. (2000). The episodic buffer: A new component of working memory? Trends in Cognitive Sciences, 4, 417-423. doi:10.1016/S1364-6613(00)01538-2

[6] Baddeley, A. D., Allen, R. J., \& Hitch, G. J. (2011). Binding in visual working memory: The role of the episodic buffer. Neuropsychologia, 49, 1393-1400. http:// dx.doi.org/10.1016/j.neuropsychologia.2010.12.042 .

[7] Botzung, A., Denkova, E., \& Manning, L. (2008). Experiencing past and future personal events: Functional neuroimaging evidence on the neural bases of mental time travel. Brain and Cognition, 66, 202-212. doi:10.1016/j.bandc.2007.07.011

[8] D'Argembeau, A., \& Van der Linden, M. (2004). Phenomenal characteristics associated with projecting oneself back into the past and forward into the future: Influence of valence and temporal distance. Consciousness and Cognition: An $\begin{array}{llrr}\text { International } & \text { Journal, } & 13, & 844-858 .\end{array}$ doi:10.1016/j.concog.2004.07.007

[9] D'Argembeau, A., Raffard, S., \& Van der Linden, M. (2008). Remembering the past and imagining the future in schizophrenia. Journal of Abnormal Psychology, 117, 247251. doi:10.1037/0021-843X.117.1.247

[10] D'Argembeau, A., Ortoleva, C., Jumentier, S., \& Van der Linden, M (2010). Component process underlying future thinking. Memory \& Cognition, 38(6), 809-819.

[11] D'Argembeau, A., \& Mathy, A. (2011). Tracking the construction of episodic future thoughts. Journal of Experimental Psychology: General, 140, 258-271. http:// dx.doi.org/10.1037/a0022581.

[12] Halpern, D.F., \& LaMay, M. L. (2000). The Smarter Sex: A Critical Review of Sex Differences in Intelligence .Educational Psychology Review, Vol. 12, No. 2.

[13] Hassabis, D., Kumaran, D., Vann, S., \& Maguire, E. (2007). Patients with hippocampal amnesia cannot imagine new experiences. Proceedings of the National Academy of Sciences of the United States of America, 104, 1726-1731. doi:10.1073/pnas.061056110

[14] Hill, P. F. \& Emery, L. J. (2013). Episodic future thought: Contributions from working memory. Consciousness and cognition, 22, 677-683.

[15] Kaufman, S. B., (2006). Sex differences in mental rotation and spatial visualization ability: Can they be accounted for by differences in working memory capacity? Intelligence, 20.

[16] Levine, B. (2004). Autobiographical memory and the self in time: Brain lesion effects, functional neuroanatomy, and lifespan development. Brain and Cognition, 55(1), 54-68. doi:10.1016/S0278-2626(03)00280-X

[17] Levine, B., Svoboda, E., Hay, J. F., Winocur, G., \& Moscovitch, M. (2002). Ageing and autobiographical memory: Dissociating episodic from semantic retrieval. Psychology and Ageing, 17, 677689.

[18] Levine, B. \& Jacques, P. (2007). Ageing and autobiographical memory of emotional and neutral events. Memory, 15(2), 129-144.

[19] Lind, S. E., \& Bowler, D. M. (2010). Episodic memory and episodic future thinking in adults with autism. Journal of Abnormal Psychology, 119, 896-905. doi:10.1037/a0020631

[20] McIsaac, H. K., \& Eich, E. (2002). Vantage point in episodic memory. Psychonomic Bulletin and Review, 9, 146-150.

[21] Nigro, G., \& Neisser, U. (1983). Point of viewin personal memories. Cognitive Psychology, 15, 467-482.

[22] Okuda, J., Fujii, T., Ohtake, H., Tsukiura, T., Tanji, K., Suzuki, K., Yamadori, A. (2003). Thinking of the future and past: The roles of the frontal pole and the medial temporal lobes. NeuroImage, 19, 1369-1380. doi:10.1016/S1053-8119(03)00179-4

[23] Robinson, J. A., \& Swanson, K. L. (1993). Field and observer modes of remembering. Memory, 1, 169-184.

[24] Schacter, D. L., \& Addis, D. R. (2007a). On the constructive episodic simulation of past and future events. Behavioral and Brain Sciences, 30, 331-332. doi:10.1017/S0140525X07002178

[25] Schacter, D. L., \& Addis, D. R. (2007b). On the constructive episodic simulation of past and future events. Behavioral and Brain Sciences, 30, 331-332.

[26] Schacter, D. L., Addis, D. R., Hassabis, D., Martin, V. C., Spreng, R. N., \& Szpunar, K. K. (2012). The future of memory: Remembering, imagining, and the brain. Neuron, 76, 677-694. http://dx.doi.org/10.1016/j.neuron.2012.11.001.

[27] Stumpf, H., and Eliot, J. (1995). Gender-related differences in spatial ability and the $\mathrm{k}$ factor of general spatial ability in a population of academically talented students. Person. Indiv. Diff. 19: 33-45.

[28] Stumpf, H., and Jackson, D. N. (1994). Gender-related differences in cognitive abilities: Evidence from a medical school admissions testing program. Person. Indi. Diff. 17: 335-344.

[29] Suddendorf, T., \& Busby, J. (2005). Making decisions with the future in mind: Developmental and comparative identification of mental time travel. Learning and Motivation, 36, 110-125. doi:10.1016/j.lmot.2005.02.010

[30] Suddendorf, T., \& Corballis, M. (2007). The evolution of foresight: What is mental time travel, and is it unique to humans? Behavioral and Brain Sciences, 30, 299-313. doi: 10.1017/S0140525X07001975

[31] Szpunar, K., Watson, J., \& McDermott, K. (2007). Neural substrates of envisioning the future. Proceedings of the National Academy of Sciences of the United States of America, 104, 642-647. doi:10.1073/pnas.0610082104

[32] Szpunar, K. K., \& McDermott, K. B. (2008a). Episodic future thought and its relation to remembering: Evidence from ratings of subjective experience. Consciousness and Cognition, 17, 330-334.

[33] Szpunar, K. K., Chan, J. K., \& McDermott, K. B. (2009). Contextual processing in episodic future thought. Cerebral Cortex, 19, 1539-1548. http://dx.doi.org/ 10.1093/cercor/bhn191.

[34] Szpunar, K. (2010). Episodic future thought: An emerging 
concept. Perspectives on Psychological Science, 5, 142-162. http://dx.doi.org/10.1177/1745691610362350.

[35] Trope, Y., \& Liberman, N. (2003). Temporal construal. Psychological Review, 110, 403-421.

[36] Tulving, E. (1985). Memory and consciousness. Canadian Psychologist, 26(1), 1-12.
[37] Viard, A., Chételat, G., Lebreton, K., Desgranges, B., Landeau, B., Sayette, V. D. L., et al (2011). Mental time travel into the past and the future in healthy aged adults: An fMRI study. Brain and Cognition, 75, 1-9.

[38] Wheeler, M. A., Stuss, D. T., \& Tulving, E. (1997). Toward a theory of episodic memory: The frontal lobes and autonoetic consciousness. Psychological Bulletin, 121, 331-354. doi:10.1037/0033-2909.121.3.331. 